\title{
A MIMO CHANNEL PERTURBATION ANALYSIS FOR ROBUST BIT LOADING
}

\author{
Michael D. Larsen* \\ Brigham Young University \\ Provo, Utah 84606
}

\author{
A. Lee Swindlehurst \\ University of California, Irvine \\ Irvine, California 92697
}

\begin{abstract}
In narrowband multiple-input multiple-output (MIMO) communication systems, when the channel state information (CSI) is known perfectly at the transmitter and the receiver, techniques such as waterfilling may use the singular value decomposition to separate the MIMO channel into independent single-input single-output subchannels. The signal-to-noise ratios of these subchannels are easily found, and, therefore, so are the subchannel bit allocations. In practice, perfect CSI is difficult to obtain. Imperfect CSI results in subchannel coupling and co-channel interference. In this paper, simple first-order expressions are presented for the signal and interference/noise powers for each subchannel for the imperfect CSI case. These expressions may be used to obtain more realistic subchannel bit allocations, allowing for fewer channel outages. Numerical simulations demonstrate the applicability and usefulness of the derived expressions.
\end{abstract}

Index Terms - MIMO systems, Singular value decomposition, Resource management, Approximation methods

\section{INTRODUCTION}

Given perfect channel state information (CSI) at the transmitter and receiver of a multiple-input multiple-output (MIMO) communications link, techniques such as waterfilling may be employed to increase throughput given a fixed transmission power by using the singular value decomposition (SVD) to separate the MIMO channel into independent single-input single-output channels, enabling interference-free data multiplexing $[1,2]$. If the statistics of the measurement noise is known at each receive antenna, it is straightforward to use the power and noise levels at each subchannel to select symbol constellations to meet particular probability of error requirements [3].

Unfortunately, it is not possible in practice to obtain perfect CSI since realizable channel estimates are formed from noisy measurements. Mobility in the communication system poses an additional challenge, since the MIMO channel

\footnotetext{
* This work was supported by the U.S. Army Research Office under the Multi-University Research Initiative (MURI) grant W911NF-04-1-0224 and by the National Science Foundation under grant CCF-0313056.
}

changes rapidly when the transmit or receive arrays are in motion. When noisy or outdated CSI is used, the MIMO subchannels become coupled resulting in subchannel power loss and interference. As a result, subchannel bit-loading levels selected using the signal and noise powers of the perfect CSI assumption may no longer meet required probability-of-error levels. In order to meet these error performance constraints, the bit loading of the subchannels may need to be reduced from the perfect-CSI levels. However, without knowledge of the power levels of the signal loss and interference induced by the subchannel coupling, it is not clear how to systematically carry out such reductions.

In this paper, we develop first-order closed-form expressions for the subchannel signal and interference powers when using imperfect CSI in SVD-based signalling schemes. These expressions allow us to (1) analyze the impact of imperfect CSI on the MIMO SVD subchannels and (2) systematically select more robust bit-loading schemes when the CSI is known to be noisy or outdated. The derivations are based on the first-order SVD perturbation analysis in [4]. Unlike previous SVD perturbation studies [5,6], the expressions presented in [4] account for the effects of the subchannel interferences between singular vectors within the signal subspace, allowing us to carry out our analysis.

The remainder of this paper will proceed as follows. In Section 2, we present the MIMO channel and signalling model. First-order expressions for the channel and interference powers are then derived in Section 3. Section 4 relates the model and derivations in the previous sections with a physical model describing array motion. In Section 5, simulations demonstrate the accuracy of the derived expressions and their usefulness when applied to the bit-loading problem.

\section{FIRST-ORDER SIGNAL MODEL}

Consider the following narrow-band MIMO SVD-based signaling scheme:

$$
\mathbf{y}=\mathbf{U}_{s}^{H}\left[(\mathbf{H}+\Delta \mathbf{H}) \mathbf{V}_{s} \mathbf{P x}+\mathbf{n}\right] .
$$

The matrix $\mathbf{H}$ is the $N_{r} \times N_{t}$ MIMO channel as known at the transmitter and receiver and $\mathbf{H}+\Delta \mathbf{H}$ is the actual MIMO channel encountered during transmission of the data vector $\mathbf{x}$. 
The matrix $\mathbf{H}$ has the following singular value decomposition (SVD):

$$
\mathbf{H}=\mathbf{U}_{s} \boldsymbol{\Sigma}_{s} \mathbf{V}_{s}^{H}+\mathbf{U}_{n} \boldsymbol{\Sigma}_{n} \mathbf{V}_{n}^{H} .
$$

The matrices $\mathbf{U}_{s}$ and $\mathbf{V}_{s}$ contain as columns the singular vectors corresponding to the nonzero singular values of $\mathbf{H}$. If we assume there are $F$ nonzero singular values, then $\mathbf{U}_{s}$ is $N_{r} \times F, \mathbf{V}_{s}$ is $N_{t} \times F$, and $\boldsymbol{\Sigma}_{s}$ is an $F \times F$ diagonal matrix with the nonzero singular values on the diagonal. In a like manner, $\mathbf{U}_{n}$ and $\mathbf{V}_{n}$ contain the singular vectors corresponding to the zero-valued singular values and $\boldsymbol{\Sigma}_{n}$ is a zero matrix. The matrix $\mathbf{P}$ is a real-valued $F \times F$ diagonal power-loading matrix whose entries determine the signal power given to the unit-norm elements of $\mathbf{x}$. The vector $\mathbf{n}$ is zero-mean measurement noise. The perturbed (actual) channel $\mathbf{H}+\Delta \mathbf{H}$ has the subspace decomposition

$$
\mathbf{H}+\Delta \mathbf{H}=(\mathbf{H}+\Delta \mathbf{H})_{s}+(\mathbf{H}+\Delta \mathbf{H})_{n}
$$

where

$$
(\mathbf{H}+\Delta \mathbf{H})_{s}=\left(\mathbf{U}_{s}+\Delta \mathbf{U}_{s}\right)\left(\boldsymbol{\Sigma}_{s}+\Delta \boldsymbol{\Sigma}_{s}\right)\left(\mathbf{V}_{s}+\Delta \mathbf{V}_{s}\right)^{H}
$$

and $(\mathbf{H}+\Delta \mathbf{H})_{n}$ is defined in a like manner. We assume that the perturbations to singular vectors are orthogonal to the singular vectors themselves, e.g., $\Delta \mathbf{u}_{f} \perp \mathbf{u}_{f}$, where $u_{f}$ is the $f$-th column of $\mathbf{U}_{s}$ and $\Delta \mathbf{u}_{f}$ is defined in a like manner. Inserting (3) into (1) and dropping higher-order terms, we find

$$
\mathbf{y} \approx\left[\boldsymbol{\Sigma}_{s}+\Delta \boldsymbol{\Sigma}_{s}+\mathbf{Q}_{1} \boldsymbol{\Sigma}_{s}+\boldsymbol{\Sigma}_{s} \mathbf{Q}_{2}^{H}\right] \mathbf{P x}+\mathbf{U}_{s}^{H} \mathbf{n}
$$

where $\mathbf{Q}_{1}=\mathbf{U}_{s}^{H} \Delta \mathbf{U}_{s}$ and $\mathbf{Q}_{2}=\Delta \mathbf{V}_{s}^{H} \mathbf{V}_{s}$. If we assume that the perturbation $\Delta \mathbf{H}$ is small enough (see [4]), then we may use the formulation in [4] to write $\mathbf{Q}_{1}, \mathbf{Q}_{2}$, and $\Delta \Sigma_{s}$ in terms of the SVD of $\mathbf{H}$ and the perturbation $\Delta \mathbf{H}$. That is,

$$
\begin{aligned}
\mathbf{Q}_{1} & =\mathbf{D} \odot\left(\mathbf{U}_{s}^{H} \Delta \mathbf{H} \mathbf{V}_{s} \boldsymbol{\Sigma}_{s}+\boldsymbol{\Sigma}_{s} \mathbf{V}_{s}^{H} \Delta \mathbf{H}^{H} \mathbf{U}_{s}\right) \\
\mathbf{Q}_{2} & =\mathbf{D} \odot\left(\mathbf{V}_{s}^{H} \Delta \mathbf{H}^{H} \mathbf{U}_{s} \boldsymbol{\Sigma}_{s}+\boldsymbol{\Sigma}_{s} \mathbf{U}_{s}^{H} \Delta \mathbf{H} \mathbf{V}_{s}\right) \\
\Delta \boldsymbol{\Sigma}_{s} & =\operatorname{diag}\left(\Delta \sigma_{1}, \Delta \sigma_{2}, \ldots, \Delta \sigma_{F}\right)
\end{aligned}
$$

where $\odot$ is the element-wise, or Hadamard, matrix product,

$$
[\mathbf{D}]_{f g}=\left\{\begin{array}{cc}
\left(\sigma_{g}^{2}-\sigma_{f}^{2}\right)^{-1}, & f \neq g \\
0, & f=g
\end{array},\right.
$$

and

$$
\Delta \sigma_{f}=\frac{1}{2}\left(\mathbf{u}_{f}^{H} \Delta \mathbf{H} \mathbf{v}_{f}+\mathbf{v}_{f}^{H} \Delta \mathbf{H}^{H} \mathbf{u}_{f}\right) .
$$

Note that in (5), the diagonal term $\Sigma_{s}+\Delta \Sigma_{s}$ corresponds to the signal portion of the overall channel while the term $\mathbf{Q}_{1} \boldsymbol{\Sigma}_{s}+\boldsymbol{\Sigma}_{s} \mathbf{Q}_{2}^{H}$, which has a diagonal of all zeros, corresponds to the interference portion of the equivalent channel.

\section{DERIVATION OF THE SINR}

Given the first-order expression for our SVD-based MIMO signalling scheme in (5), we now proceed to derive persubchannel signal and interference power expressions. Assume distributions on $\mathrm{x}$ and $\Delta \mathbf{H}$ such that $\mathrm{x}$ and $\Delta \mathbf{H}$ are independent, $\mathbf{x}$ is zero mean with covariance $\mathbf{C}_{x}$, and $\Delta \mathbf{H}$ is zero-mean circular-symmetric complex Gaussian with covariance $\mathbf{C}_{\Delta \mathbf{H}}$. Let $y_{s, i}$ be the $i$-th element of the signal portion of $\mathbf{y}$ and $\mathbf{e}_{i}$ be the $i$-th column of the identity matrix. Then the average signal power at the $i$-th receive antenna is given by

$$
\begin{aligned}
P_{s, i} & =E\left[y_{s, i} y_{s, i}^{*}\right] \\
& =E\left[\left|\mathbf{e}_{i}^{H}\left(\boldsymbol{\Sigma}_{s}+\Delta \boldsymbol{\Sigma}_{s}\right) \mathbf{P} \mathbf{x}\right|^{2}\right] \\
& =E\left[\left(\boldsymbol{\Sigma}_{s}+\Delta \boldsymbol{\Sigma}_{s}\right) \mathbf{P} \mathbf{C}_{x} \mathbf{P}\left(\boldsymbol{\Sigma}_{s}+\Delta \boldsymbol{\Sigma}_{s}\right)^{H}\right]_{i i} \\
& =\left[\boldsymbol{\Sigma}_{s} \mathbf{P} \mathbf{C}_{x} \mathbf{P} \boldsymbol{\Sigma}_{s}\right]_{i i}+E\left[\Delta \boldsymbol{\Sigma}_{s} \mathbf{P} \mathbf{C}_{x} \mathbf{P} \Delta \boldsymbol{\Sigma}_{s}\right]_{i i}
\end{aligned}
$$

where $[\mathbf{A}]_{f g}$ indicates the $(f, g)$-th element of $\mathbf{A}$. Note that $E\left[\Delta \boldsymbol{\Sigma}_{s}\right]=0$ since $E[\Delta \mathbf{H}]=0$ by assumption, leading to (13). Let $\mathbf{S}=E\left[\Delta \boldsymbol{\Sigma}_{s} \mathbf{P} \mathbf{C}_{x} \mathbf{P} \Delta \boldsymbol{\Sigma}_{s}\right]$. Using (10) and the circular symmetry of $\Delta \mathbf{H}$, we find after a little manipulation that

$$
\begin{aligned}
2[\mathbf{S}]_{i i} & =\left[\mathbf{P C}_{x} \mathbf{P}\right]_{i i} E\left[\left|\mathbf{u}_{i}^{H} \Delta \mathbf{H v}_{i}\right|^{2}\right] \\
& =\left[\mathbf{P C}_{x} \mathbf{P}\right]_{i i} E\left[\left|\left(\mathbf{v}_{i}^{T} \otimes \mathbf{u}_{i}^{H}\right) \operatorname{vec}(\Delta \mathbf{H})\right|^{2}\right] \\
& =\left[\mathbf{P C}_{x} \mathbf{P}\right]_{i i}\left(\mathbf{v}_{i}^{T} \otimes \mathbf{u}_{i}^{H}\right) \mathbf{C}_{\Delta \mathbf{H}}\left(\mathbf{v}_{i}^{*} \otimes \mathbf{u}_{i}\right)
\end{aligned}
$$

where $\otimes$ is the matrix Kronecker product operator and $\operatorname{vec}(\mathbf{A})$, the vectorization operator, stacks the columns of $\mathbf{A}$. Equations (13) and (16) together give us $P_{s, i}$. If $\mathbf{C}_{\Delta \mathbf{H}}=\sigma_{H}^{2} \mathbf{I}$ and $\mathbf{C}_{x}=\mathbf{I}$, then this reduces to

$$
P_{s, i}=\left(\sigma_{i}^{2}+\frac{\sigma_{H}^{2}}{2}\right)[\mathbf{P}]_{i i}^{2} .
$$

In a similar manner as above, the interference and noise power on the $i$-th subchannel maybe written as

$$
\begin{aligned}
P_{I N, i}= & E\left[\left|\mathbf{e}_{i}^{H}\left(\mathbf{Q}_{1} \boldsymbol{\Sigma}_{\mathbf{s}}+\boldsymbol{\Sigma}_{\mathbf{s}} \mathbf{Q}_{2}^{H}\right) \mathbf{P} \mathbf{x}\right|^{2}\right] \\
& +E\left[\left|\mathbf{e}_{i}^{H} \mathbf{U}_{s}^{H} \mathbf{n}\right|^{2}\right] \\
= & E\left[\mathbf{Q}_{1} \boldsymbol{\Sigma}_{s} \mathbf{P} \mathbf{C}_{x} \mathbf{P} \boldsymbol{\Sigma}_{\mathbf{s}} \mathbf{Q}_{1}^{H}\right. \\
& +2 \operatorname{Re}\left(\mathbf{Q}_{1} \boldsymbol{\Sigma}_{s} \mathbf{P} \mathbf{C}_{x} \mathbf{P} \mathbf{Q}_{2} \boldsymbol{\Sigma}_{\mathbf{s}}\right) \\
& \left.+\boldsymbol{\Sigma}_{\mathbf{s}} \mathbf{Q}_{2}^{H} \mathbf{P} \mathbf{C}_{x} \mathbf{P} \mathbf{Q}_{2} \boldsymbol{\Sigma}_{\mathbf{s}}\right]_{i i}+\mathbf{u}_{i} \mathbf{C}_{n} \mathbf{u}_{i} \\
= & E\left[a_{1, i}+2 \operatorname{Re}\left(a_{2, i}\right)+a_{3, i}\right]+\mathbf{u}_{i}^{H} \mathbf{C}_{n} \mathbf{u}_{i}
\end{aligned}
$$

where $\mathbf{C}_{n}$ is the measurement noise covariance. Note that matrices $\mathbf{Q}_{1}$ and $\mathbf{Q}_{2}$ are skew symmetric. Using this property, the circular symmetry of $\Delta \mathbf{H}$, and properties of the trace, we find after some manipulation that

$$
\begin{aligned}
& E\left[a_{1, i}\right]=\operatorname{tr}\left[\mathbf{N}_{1, i}\left(\boldsymbol{\Sigma}_{s}^{2} \mathbf{P} \mathbf{C}_{x} \mathbf{P} \boldsymbol{\Sigma}_{s}^{2}\right)\right]+\sigma_{i}^{2}\left[\mathbf{N}_{0}\right]_{i i} \\
& E\left[a_{2, i}\right]=-\operatorname{tr}\left[\mathbf{N}_{2, i}\left(\boldsymbol{\Sigma}_{s}^{2} \mathbf{P} \mathbf{C}_{x} \mathbf{P}\right)\right]-\sigma_{i}^{2}\left[\mathbf{N}_{0}\right]_{i i} \\
& E\left[a_{3, i}\right]=\operatorname{tr}\left[\mathbf{N}_{3, i}\left(\mathbf{P} \mathbf{C}_{x} \mathbf{P}\right)\right]+\sigma_{i}^{2}\left[\mathbf{N}_{0}\right]_{i i}
\end{aligned}
$$

where for $f \neq i$ and $g \neq i$

$$
\begin{aligned}
& {\left[\mathbf{N}_{1, i}\right]_{f g}=\frac{\mathbf{u}_{i}^{H}\left(\mathbf{v}_{g}^{T} \otimes \mathbf{I}\right) \mathbf{C}_{\Delta \mathbf{H}}\left(\mathbf{v}_{f}^{*} \otimes \mathbf{I}\right) \mathbf{u}_{i}}{\left(\sigma_{f}^{2}-\sigma_{i}^{2}\right)\left(\sigma_{g}^{2}-\sigma_{i}^{2}\right)}} \\
& {\left[\mathbf{N}_{2, i}\right]_{f g}=\sigma_{i}^{2}\left[\mathbf{N}_{1, i}\right]_{f g}} \\
& {\left[\mathbf{N}_{3, i}\right]_{f g}=\sigma_{i}^{4}\left[\mathbf{N}_{1, i}\right]_{f g}}
\end{aligned}
$$


and $\left[\mathbf{N}_{1, i}\right]_{f g}=\left[\mathbf{N}_{2, i}\right]_{f g}=\left[\mathbf{N}_{3, i}\right]_{f g}=0$ if $f=i$ or $g=i$. The expression for $\mathbf{N}_{0}$ is more involved, but the diagonal elements are real-valued. Therefore, $\mathbf{N}_{0}$ does not appear in the final expression of $P_{I N, i}$, so we do not include it here. With (21)-(26), we now have an expression for $P_{I N, i}$. If $\mathbf{C}_{\Delta \mathbf{H}}=\sigma_{H}^{2} \mathbf{I}, \mathbf{C}_{x}=\mathbf{I}$, and $\mathbf{C}_{n}=\sigma_{n}^{2} \mathbf{I}$, then this reduces to

$$
P_{I N, i}=\sigma_{H}^{2} \sum_{\substack{f=1 \\ f \neq i}}^{F}[\mathbf{P}]_{f f}^{2}+\sigma_{n}^{2} .
$$

Together, (17) and (27) constitute a first-order average signalto-interference-and-noise ratio (SINR) approximation.

\section{THE GAUSS-MARKOV MODEL}

When the source of the CSI imperfection is motion, it may be instructive to relate the average power of the perturbation $\Delta \mathbf{H}$ to physical parameters describing the array motion. A simple example of doing so involves use of a Gauss-Markov fading channel model [7], where the current channel $\mathbf{H}_{t}$ is related to a reference channel $\mathbf{H}_{t-r}$ according to the following firstorder auto-regressive (AR) model

$$
\mathbf{H}_{t}=\sqrt{\alpha_{r}} \mathbf{H}_{t-r}+\sqrt{1-\alpha_{r}} \mathbf{E}_{t}
$$

where $\mathbf{E}_{t}$ is independent of $\mathbf{H}_{t-r}$ with $\operatorname{vec}\left(\mathbf{H}_{t-r}\right), \operatorname{vec}\left(\mathbf{E}_{t}\right) \sim$ $\mathbb{C N}(\mathbf{0}, \mathbf{I})$ and $0 \leq \alpha_{r} \leq 1$. The parameter $\alpha_{r}$ may be related to the physical propagation environment by matching it to the second-order statistics associated with an appropriate propagation model. For example, when using Jakes' channel model [8], it is shown in [7] that $\alpha_{r}$ may be chosen as

$$
\alpha_{r}=J_{0}(2 \pi r f)^{2}
$$

where $J_{0}(\cdot)$ is the zeroth-order Bessel function of the first kind, $f=f_{d} T_{s}, f_{d}$ is the maximum Doppler frequency in the fading environment, and $T_{s}$ is the sampling period. It is straight forward to connect the model in (28) with that used in Section 2. Following this connection through the derivations in Section 3, we find

$$
\begin{gathered}
P_{s, i}=\left(\alpha_{r} \sigma_{i}^{2}+\frac{1-\alpha_{r}}{2}+\frac{\sigma_{H, \mathbf{n}}^{2}}{2}\right)[\mathbf{P}]_{i i}^{2} \\
P_{I N, i}=\left(1-\alpha_{r}+\sigma_{H, \mathbf{n}}^{2}\right) \sum_{\substack{f=1 \\
f \neq i}}^{F}[\mathbf{P}]_{f f}^{2}+\sigma_{n}^{2}
\end{gathered}
$$

where $\sigma_{H, \mathbf{n}}^{2}$ is the channel perturbation variance due to the measurement noise.

\section{NUMERICAL RESULTS}

The expressions in (17) and (27) (or (30) and (31)) are first order in the channel perturbations. We begin our numerical



Fig. 1. A comparison of the $1^{\text {st }}$-order subchannel SINR approximation with Monte Carlo SINR results.

simulations by investigating the accuracy of these expressions through simulation. This will be done by comparing our average first-order subchannel SINR approximations with SINR values generated through Monte Carlo methods. In particular, we assume a $4 \times 4 \mathrm{MIMO}$ channel $\mathbf{H}$ selected such that $\operatorname{vec}(\mathbf{H}) \sim \mathbb{C N}\left(\mathbf{0}, \mathbf{I}_{16}\right)$. The waterfilling solution is used to select the subchannel power levels, and we assume a measurement noise variance of $-20 \mathrm{~dB}$ relative to unity. Using this setup and the power and interference expressions given above, we find SINR values for the first three MIMO subchannels for several different channel perturbation powers, and compare these results to those generated through Monte Carlo methods assuming vec $(\Delta \mathbf{H}) \sim \mathbb{C N}\left(\mathbf{0}, \sigma_{H}^{2} \mathbf{I}\right)$. The results of this simulation, averaged over 1000 channel realizations, are displayed in Fig. 1. It is clear from these results that the proposed first-order expressions provide SINR estimates with good accuracy, even for channel channel perturbations on the same order as the channel itself.

One possible application of these first-order power and interference expressions is the problem of subchannel bit loading, which we explore in the next simulation. Consider the task of subchannel bit loading using the power levels dictated by the waterfilling solution. To ensure reliable communication, we require that the symbol error rate (SER) of each subchannel remain below an SER threshold of 0.01. If a subchannel exceeds that level, we say a channel outage has occurred. Assuming a similar simulation setup as above and using the QAM constellations and SER expressions in [3], constellations sizes are chosen that result in SER-constraint-meeting bit rates. Note that a margin of safety is included when determining the constellation sizes to prevent excessive outages. When bit loading using the power levels given by waterfilling, we expect high bit rates, but a large number of outages may occur when the CSI is poor. 


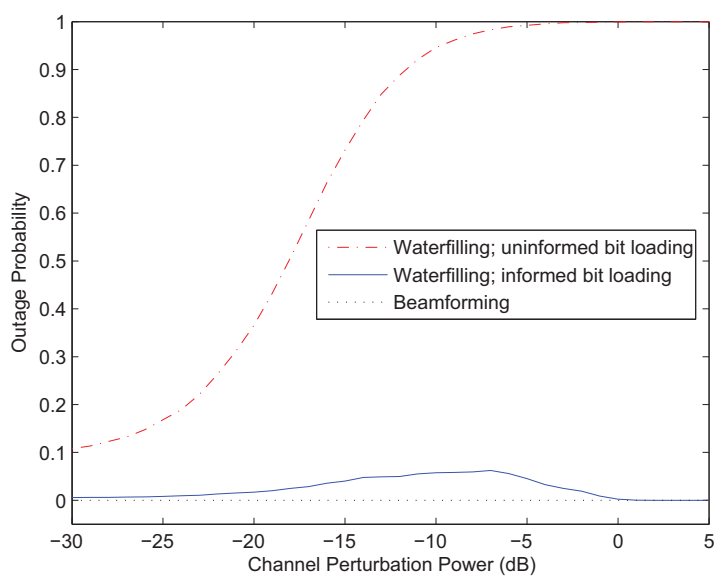

Fig. 2. A comparison of waterfilling outage probabilities using the uninformed and informed bit-loading schemes.

To reduce the number of outages, we propose an informed bit-loading method that assigns bits based upon knowledge of the channel perturbation power and the expressions in (17) and (27). If a subchannel cannot support any bits, the waterfilling power levels are modified so that the subchannel's allocated power will be redistributed to the other subchannels proportionally. Figure 2 compares outage probabilities (determined through Monte Carlo simulation) of waterfilling for the uninformed and informed bit-loading strategies over a range of motion-related channel perturbation powers. Also included are results for beamforming using the dominant left and right singular vectors with perturbation-aware bit loading. The initial outage levels on the left of the plot are a result of measurement-noise-induced channel perturbations, and the outage percentages change as motion-induced perturbations increase. The outage level of informed bit loading eventually decreases, approaching that of beamforming, as this method reduces to the beamforming solution. It is clear that the informed bit loading method results in significantly lower outage percentages than the uninformed method realizes.

If we assume bits corresponding to outages are lost, we may plot the effective average bits per transmission of the bit-loading schemes. Figure 3 compares the average bits per transmission of waterfilling using both uninformed and informed bit loading, and beamforming, for a range of channel perturbation powers. In the figure, waterfilling with informed bit loading demonstrates better overall throughput than the uninformed method over a wide range of channel perturbation powers. This improved performance is made possible by the proposed subchannel power and interference expressions. The results also indicate what level of channel estimation inaccuracies is tolerable before beamforming should replace our data multiplexing schemes. This is another benefit of our analysis.

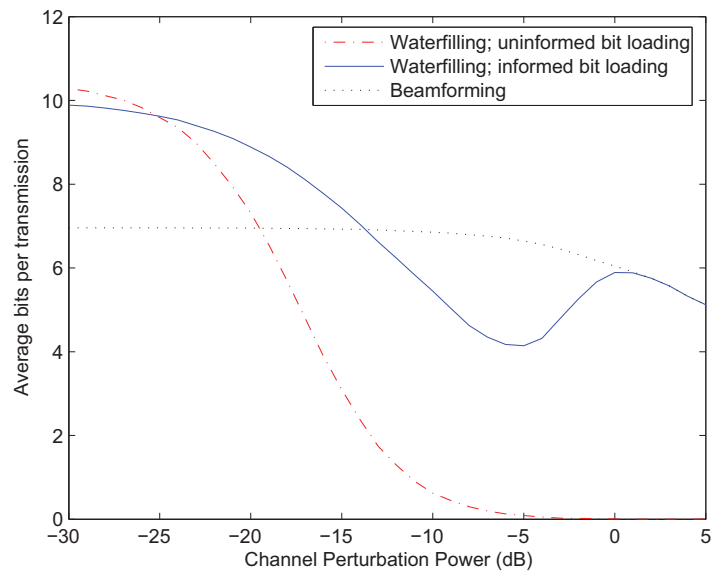

Fig. 3. A comparison of the effective average bits per transmission achieved using waterfilling with uninformed and informed bit loading and beamforming.

\section{REFERENCES}

[1] I. E. Telatar, "Capacity of multi-antenna gaussian channels,” AT\&T Bell Labs, Internal Tech. Memo., Jun. 1995.

[2] G. J. Foschini and M. J. Gans, "On limits of wireless communications in a fading environment when using multiple antennas," Wireless Pers. Commun., vol. 6, pp. 311-335, Mar. 1998.

[3] J. G. Proakis, Digital Communications, 4th ed. New York, NY: McGraw-Hill, 2001.

[4] J. Liu, X. Liu, and X. Ma, "First-order perturbation analysis of singular vectors in the singular value decomposition," IEEE Trans. Signal Process., vol. 56, pp. 30443049, Jul. 2008.

[5] B. Friedlander and A. J. Weiss, "On the second-order statistics of the eigenvectors of sample covariance matrices," IEEE Trans. Signal Process., vol. 46, pp. 3136 3139, Nov. 1998.

[6] Z. Xu, "Perturbation analysis for subspace decomposition with applications in subspace-based algorithms," IEEE Trans. Signal Process., vol. 50, pp. 2820-2830, Nov. 2002.

[7] C. B. Peel and A. L. Swindlehurst, "Performance of space-time modulation for a generalized time-varying rician channel model," IEEE Trans. Wireless Commun., vol. 3, pp. 1003-1012, May 2004.

[8] W. C. Jakes, Microwave Mobile Communications. New York, NY: Wiley-IEEE Press, 1994. 